Research Article

\title{
The Effects of Harvesting on the Dynamics of a Leslie-Gower Model
}

\author{
Jingli Xie $\mathbb{D}^{1},{ }^{1}$ Hanyan Liu $\mathbb{D}^{1},{ }^{1}$ and Danfeng Luo $\mathbb{D}^{2}$ \\ ${ }^{1}$ College of Mathematics and Statistics, Jishou University, Jishou 416000, Hunan, China \\ ${ }^{2}$ Department of Mathematics, Guizhou University, Guiyang 550025, Guizhou, China
}

Correspondence should be addressed to Hanyan Liu; liuhanyan1998@163.com

Received 15 January 2021; Accepted 24 April 2021; Published 7 May 2021

Academic Editor: Rigoberto Medina

Copyright (c) 2021 Jingli Xie et al. This is an open access article distributed under the Creative Commons Attribution License, which permits unrestricted use, distribution, and reproduction in any medium, provided the original work is properly cited.

In this paper, we study a Leslie-Gower predator-prey model with harvesting effects. We carry out local bifurcation analysis and stability analysis. Under certain conditions, the model is shown to undergo a supercritical Hopf bifurcation resulting in a stable limit cycle. Numerical simulations are presented to illustrate our theoretic results.

\section{Introduction}

In this paper, we consider Leslie-Gower predator-prey model with harvesting effect,

$$
\left\{\begin{array}{l}
\frac{\mathrm{d} x_{1}}{\mathrm{~d} \tau}=r x_{1}\left(1-\frac{x_{1}}{k}\right)-\frac{a_{1} x_{1} x_{2}}{n+x_{1}}-e_{1} x_{1}, \\
\frac{\mathrm{d} x_{2}}{\mathrm{~d} \tau}=s x_{2}\left(1-\frac{a_{2} x_{2}}{n+x_{1}}\right)-e_{2} x_{2},
\end{array}\right.
$$

with $x_{1}(0) \geq 0$ and $x_{2}(0) \geq 0$, where $x_{1}(\tau)$ and $x_{2}(\tau)$ are the prey and predator population densities, respectively, $r, s, a_{1}, a_{2}, n, e_{1}, e_{2}>0$, and $\tau$ is the time.

Note that $\left(a_{2} x_{2} /\left(n+x_{1}\right)\right)$ is Leslie-Gower term in which the carrying capacity of the predator's environment is a linear function of the prey size $\left(x_{1} / a_{2}\right)+\left(n / a_{2}\right)$. $\left(a_{1} x_{1} x_{2} /\left(n+x_{1}\right)\right)$ is the number of prey consumed by the predator in unit time which shows that when the number of the prey $x_{1}$ is severe scarcity, and the predators can switch over to other populations as food. Constants $r$ and $s$ are the intrinsic growth rate of the prey and predator, respectively, and $e_{1}$ and $e_{2}$ denote the harvesting efforts for the prey and predator, respectively.

Since the first prey-predator dynamical models which is the Lotka-Volterra model was built in the 1920s by
Mathematician Lotka and Volterra, more and more researchers are interested in such issues, and they start from different angles to think the problem and many important results have been obtained [1-10]. In particular, in 2003, Aziz-Alaoui and Daher Okiye [11] considered the following Leslie-Gower predator-prey model:

$$
\left\{\begin{array}{l}
\frac{\mathrm{d} x_{1}}{\mathrm{~d} \tau}=r x_{1}\left(1-\frac{x_{1}}{k}\right)-\frac{a_{1} x_{1} x_{2}}{n_{1}+x_{1}}, \\
\frac{\mathrm{d} x_{2}}{\mathrm{~d} \tau}=s x_{2}\left(1-\frac{a_{2} x_{2}}{n_{2}+x_{1}}\right),
\end{array}\right.
$$

where $x_{1}$ is the numbers of prey and $x_{2}$ is the numbers of predators. Existence and stability of the fixed points were studied by using the Lyapunov function. In 2006, Lin and Ho [12] discussed the local and global stability for system (2) by using Poincaré-Bendixson theorem and Dulac's criterion.

Harvesting is an effective way for humans to control the size of predators and prey so that the population has continued to develop healthily and produced good economic benefits [13-16]. Academically, researchers often only consider the harvesting of prey in order to control the size of the population. In 2010, Zhu and Lan [17] investigated the Leslie-Gower predator-prey systems: 


$$
\left\{\begin{array}{l}
\frac{\mathrm{d} x_{1}}{\mathrm{~d} \tau}=r_{1} x_{1}\left(1-\frac{x_{1}}{k}\right)-a x_{1} x_{2}-h, \\
\frac{\mathrm{d} x_{2}}{\mathrm{~d} \tau}=r_{2} x_{2}\left(1-\frac{x_{2}}{b x_{1}}\right) .
\end{array}\right.
$$

In 2013, Gupta and Chandra [18] discussed the following Leslie-Gower predator-prey model with harvesting on the prey and the environment providing the same protection to both the predator and prey:

$$
\left\{\begin{array}{l}
\frac{\mathrm{d} x_{1}}{\mathrm{~d} \tau}=r x_{1}\left(1-\frac{x_{1}}{k}\right)-\frac{a_{1} x_{1} x_{2}}{n+x_{1}}-\frac{q E x_{1}}{m_{1} E+m_{2} x_{1}}, \\
\frac{\mathrm{d} x_{2}}{\mathrm{~d} \tau}=s x_{2}\left(1-\frac{a_{2} x_{2}}{n+x_{1}}\right) .
\end{array}\right.
$$

For ecological balance and healthy economic development, for fisheries, wildlife resources, etc., we not only need to consider the harvesting of prey, but also the predator. Therefore, in this paper, we study Leslie-Gower predatorprey model (1) with harvesting on the prey and predator.

In order to make the system dimensionless, we define new dependent variables by $x=\left(x_{1} / k\right), y=\left(\left(a_{1} x_{2}\right) / k\right)$ and a new time variable by $t=r \tau$. Moreover, let $\alpha=(1 / r)$, $\beta=\left(a_{2} / a_{1}\right), \quad m=(n / k), \quad \rho=(s / r), \quad \gamma=\left(e_{1} / r\right), \quad$ and $\delta=\left(e_{2} /\left(r a_{1}\right)\right)$. System (1) becomes

$$
\left\{\begin{array}{l}
\frac{\mathrm{d} x}{\mathrm{~d} t}=x(1-x)-\frac{\alpha x y}{m+x}-\gamma x \\
\frac{\mathrm{d} y}{\mathrm{~d} t}=\rho y\left(1-\frac{\beta y}{m+x}\right)-\delta y
\end{array}\right.
$$

where $\alpha, \beta, \gamma, \rho, \delta$, and $m$ are all positive parameters.

Lemma 1 (see 19). If $a>0, b>0$, and $\dot{x} \leq x\left(b-a x^{\alpha}\right)$, where $\alpha$ is a positive constant, $t \geq 0$, and $x(0)>0$, we have

$$
x(t) \leq\left(\frac{b}{a}\right)^{(1 / \alpha)}\left(1+\left(\frac{b x^{-\alpha}(0)}{a}-1\right) e^{-b \alpha t}\right)^{-(1 / \alpha)} .
$$

Lemma 2 (see $[20,21])$. Consider system $\dot{X}=f(X, \alpha)$ and suppose that $f\left(X_{0}, \alpha_{0}\right)=0, n \times n$ Jacobian matrix $\left(J \equiv D f\left(X_{0}, \alpha_{0}\right)\right)$ has a simple eigenvalue $s=0$ with eigenvector $V$, and the transpose of the Jacobian matrix $J^{T}$ has an eigenvector $W$ to the eigenvalue $s=0$. Then, the system $\dot{X}=f(X, \alpha)$ experiences a transcritical bifurcation at the equilibrium point $X_{0}$ as the control parameter $\alpha$ passes through the bifurcation value $\alpha=\alpha_{0}$ if the following conditions are satisfied:

$$
\begin{aligned}
W^{T} D f_{\alpha}\left(X_{0}, \alpha_{0}\right) & =0, \\
W^{T}\left(D f_{\alpha}\left(X_{0}, \alpha_{0}\right) V\right) & \neq 0, \\
W^{T}\left(D^{2} f\left(X_{0}, \alpha_{0}\right)(V, V)\right) & =0 .
\end{aligned}
$$

The rest of this paper is organized as follows. In Section 2, we study boundary of solutions. In Section 3, we discuss existence of equilibria points. In Section 4, we discuss stability of the equilibrium points.

\section{Boundedness of Solutions}

In this section, we prove that every solution of system (5) is positive and uniformly bounded with initial conditions $x(0)=x_{0}>0 \quad$ and $\quad y(0)=y_{0}>0 . \quad$ Denote $\mathbb{R}_{+}^{2}=\left\{(x(t), y(t)) \in \mathbb{R}^{2} \mid x(t)>0, y(t)>0, t>0\right\}$.

Theorem 1. Consider system (5). For any given initial conditions $\left(x_{0}, y_{0}\right) \in \mathbb{R}_{+}^{2}$, the solution $(x(t), y(t))$ of system (5) exists and is unique and positive and ultimate bounded.

Proof. Let function $f(x \quad(t), y(t))=(x(t)(1-x$ $(t))-(\alpha x(t) y(t) /(m+x(t)))-\gamma x(t), \rho y(t)(1-(\beta y$ $(t) /(m+x(t))))-\delta y(t))$ Obviously, function $f(x(t), y(t))$ is continuous differentiable on $(x, y) \in \mathbb{R}_{+}^{2}$, so for any given the initial conditions $\left(x_{0}, y_{0}\right) \in \mathbb{R}_{+}^{2}$, the solution $(x(t), y(t))$ of the system (5) exists and is unique. Furthermore, $x$-axis and $y$-axis are the solutions of the system (5); by the uniqueness of the solution, the solutions $(x(t), y(t))$ of the system (5) with the initial value $x_{0}>0, y_{0}>0$ cannot cross with $x$-axis and $y$-axis.

Next, we show the solutions $(x(t), y(t))$ of system (5) with the initial value $x_{0}>0$ and $y_{0}>0$ which is ultimate bounded.

From system (5), we have

$$
\frac{\mathrm{d} x}{\mathrm{~d} t} \leq x(1-x)
$$

Combining Lemma 1, we have

$$
\begin{aligned}
x(t) & \leq\left(1+\left(x^{-1}(0)-1\right) e^{-t}\right)^{-1} \leq M_{0}, \\
M_{0} & =\max \left\{1, x_{0}\right\} .
\end{aligned}
$$

So, the following inequality is established:

$$
\frac{\mathrm{d} y}{\mathrm{~d} t} \leq \rho y\left(1-\frac{\beta y}{m+x}\right) \leq \rho y\left(1-\frac{\beta y}{m+1}\right)=y\left(\rho-\frac{\rho \beta y}{m+1}\right) \text {. }
$$

By Lemma 1, we have

$$
y(t) \leq M_{1}, \quad t \geq 0
$$

where $M_{1}=\max \left\{\left(\left(m+M_{0}\right) / \beta\right), y_{0}\right\}$.

\section{Existence of Equilibria}

In order to find the equilibrium points of system (5), we let $f(x(t), y(t))=0$, i.e.,

$$
\left\{\begin{array}{c}
x(1-x)-\frac{\alpha x y}{m+x}-\gamma x=0, \\
\rho y\left(1-\frac{\beta y}{m+x}\right)-\delta y=0 .
\end{array}\right.
$$


It is clear that equation (12) has a trivial solution $E_{0}:=(0,0)$. Furthermore, by calculation, we find other solutions of equation (12):

$$
\begin{aligned}
& (1-\gamma, 0), \\
& \left(0, \frac{(\rho-\delta) m}{\rho \beta}\right), \\
& \left(\frac{(1-\gamma) \rho \beta-(\rho-\delta) \alpha}{\rho \beta}, \frac{(\rho-\delta)((1+m-\gamma) \rho \beta-(\rho-\delta) \alpha)}{(\rho \gamma)^{2}}\right) .
\end{aligned}
$$

Remark 1. When $\gamma<1, \delta<\rho$ and $(\rho-\delta) \alpha<(1-\gamma) \rho \beta$, the position of each point in (13) is shown in Figure 1.

Therefore, we have the following results.where $x_{\infty}=(((1-\gamma) \rho \beta-$

$(\rho-\delta) \alpha) / \rho \beta), y_{\infty}=(((\rho-\delta)((1+m-\gamma) \rho \beta-(\rho-\delta) \alpha))$ $\left./(\rho \beta)^{2}\right)$.

Theorem 2. Consider system (5) admits $x$-axial only and $y$-axial equilibria under following conditions.

(i) The $x$-axial equilibrium,

$$
E_{1}:=(K, 0), \quad \text { with } K=1-\gamma,
$$

is a boundary equilibrium of system (5) if and only if

$$
1-\gamma>0 .
$$

(ii) The $y$-axial equilibrium,

$$
E_{2}:=(0, M), \quad \text { with } M=\frac{(\rho-\delta) m}{\rho \beta},
$$

is a boundary equilibrium of system (5) if and only if

$$
\rho-\delta>0 .
$$

Theorem 3. Consider system (5) admits a unique positive equilibrium,

$$
E_{3}:=\left(x_{\infty}, y_{\infty}\right) \text {, }
$$

if only if

$$
\frac{1-\gamma}{\alpha}>\frac{\rho-\delta}{\rho \beta}>0
$$

Remark 2. Existence regions for equilibrium points of system (12) is shown in Figure 2. Equilibrium points $E_{1}$ and $E_{2}$ exist, but positive equilibrium point $E_{3}$ does not in region II and equilibrium points $E_{1}, E_{2}$, and $E_{3}$ coexist in region I. Moreover, if $((1-\gamma) / \alpha)=((\rho-\delta) / \rho \beta)>0$, then positive equilibrium $E_{3}$ becomes boundary equilibrium $E_{2}$.

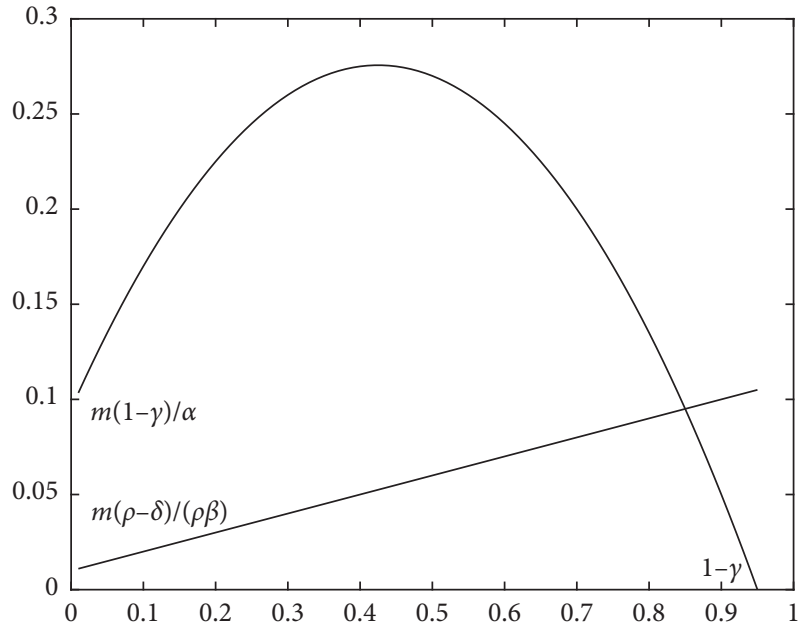

FIgURE 1: The position of each point in (13) with $\rho=0.1, \alpha=1$, $\delta=0.05, \gamma=0.05, \beta=5$, and $m=0.1$.

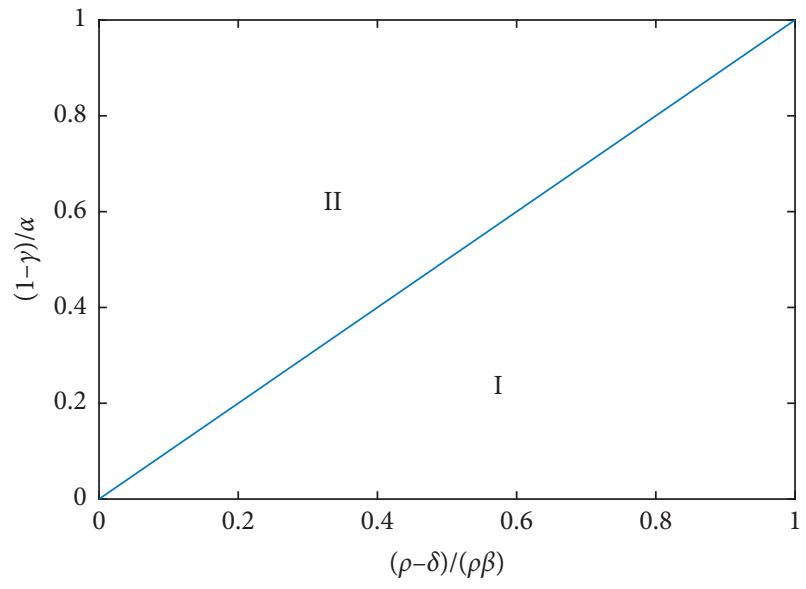

FIGURE 2: Regions for equilibrium points of system (12).

\section{Stability of of Equilibria}

Theorem 4. If $1-\gamma>0$ and $\rho-\delta>0$, then the equilibria $E_{0}$ and $E_{1}$ are unstable.

Proof. Firstly, we show the equilibria $E_{0}$ is a unstable equilibrium. The Jacobian matrix about $E_{0}$ is given by

$$
J_{E_{0}}=\left(\begin{array}{cc}
1-\gamma & 0 \\
0 & \rho-\delta
\end{array}\right)
$$

with trace $1-\gamma+\rho-\delta$ and determinant $(1-\gamma)(\rho-\delta)$. From $1-\gamma>0$ and $\rho-\delta>0$, we have $1-\gamma+\rho-\delta>0$ and $(1-\gamma)(\rho-\delta)>0$. On the contrary, through direct calculation, we obtain $(1-\gamma+\rho-\delta)^{2}-4(1-\gamma)(\rho-\delta)=$ $(1-\gamma-\rho+\delta)^{2}>0$. Therefore, $E_{0}$ is an unstable node point.

Secondly, we show the equilibria $E_{1}$ is a unstable equilibrium. The Jacobian matrix about $E_{1}$ is given by 


$$
J_{E_{1}}=\left(\begin{array}{cc}
-(1-\gamma) & -\frac{\alpha(1-\gamma)}{m+1-\gamma} \\
0 & \rho-\delta
\end{array}\right) \text {, }
$$

with determinant $-(1-\gamma)(\rho-\delta)<0$. Therefore, $E_{1}$ is a saddle point which is unstable.

Theorem 5. (1) If $((1-\gamma) / \alpha)>((\rho-\delta) / \rho \beta)>0$, then the boundary equilibrium $E_{2}$ is a unstable equilibrium. (2) If $0<((1-\gamma) / \alpha)<((\rho-\delta) / \rho \beta)$, then the boundary equilibrium $E_{2}$ is locally asymptotically stable.

Proof. The Jacobian matrix about $E_{2}$ is given by

$$
J_{E_{2}}=\left(\begin{array}{cc}
(1-\gamma)-\frac{\alpha(\rho-\delta)}{\rho \beta} & 0 \\
\frac{(\rho-\delta)^{2}}{\rho \beta} & -(\rho-\delta)
\end{array}\right) \text {, }
$$

with trace $(1-\gamma)-((\alpha(\rho-\delta)) / \rho \beta)-(\rho-\delta)$ and determinant $-(\rho-\delta)((1-\gamma)-((\alpha(\rho-\delta)) / \rho \beta))$.

(1) When $((1-\gamma) / \alpha)>((\rho-\delta) / \rho \beta)>0$, we have $-(((\rho-\delta)(\rho \beta(1-\gamma)-\alpha(\rho-\delta))) / \rho \beta)<0$, so $E_{2}$ is a saddle point which is an unstable.

(2) When $0<((1-\gamma) / \alpha)<((\rho-\delta) / \rho \beta)$, we have $(1-\gamma)-((\alpha(\rho-\delta)) / \rho \beta)-(\rho-\delta)=((\rho \beta(1-\gamma)-$ $\alpha(\rho-\delta)) / \rho \beta)-(\rho-\delta)<0$

and $-(((\rho-\delta)(\rho \beta(1-\gamma)-\alpha(\rho-\delta))) / \rho \beta)>0$. Furthermore, a direct calculations gives

$$
\begin{array}{r}
\left((1-\gamma)-\frac{\alpha(\rho-\delta)}{\rho \beta}-(\rho-\delta)\right)^{2}+4(\rho-\delta)\left((1-\gamma)-\frac{\alpha(\rho-\delta)}{\rho \beta}\right) \\
=\left((1-\gamma)-\frac{\alpha(\rho-\delta)}{\rho \beta}+(\rho-\delta)\right)^{2}>0 .
\end{array}
$$

Therefore, $E_{2}$ is locally asymptotically stable.
Theorem 6. (1) If $0<((1-\gamma) / \alpha)<((\rho-\delta) / \rho \beta)$ and $m<\left(\left(-2 x_{\infty}^{2}+(1-\gamma-\rho+\delta) x_{\infty}\right) /\left(x_{\infty}+\rho-\delta\right)\right)$ hold, then the positive equilibrium $E_{3}$ is unstable. (2) If $0<((1-\gamma) / \alpha)<((\rho-\delta) / \rho \beta)$ and $m>\left(\left(-2 x_{\infty}^{2}+(1-\gamma-\rho+\delta) x_{\infty}\right) /\left(x_{\infty}+\rho-\delta\right)\right)$ hold, then the positive equilibrium $E_{3}$ is locally asymptotically stable. Furthermore, assume that there is $\alpha<\rho \beta$; then, the positive equilibrium $E_{3}$ is globally asymptotically stable.

Proof. The Jacobian matrix about $E_{3}$ is given by

$$
J_{E_{3}}=\left(\begin{array}{cc}
x_{\infty}\left(-1+\frac{1-\gamma-x_{\infty}}{m+x_{\infty}}\right) & -\frac{\alpha x_{\infty}}{m+x_{\infty}} \\
\frac{(\rho-\delta)^{2}}{\rho \beta} & -(\rho-\delta)
\end{array}\right) \text {, }
$$

with trace $x_{\infty}\left(-1+\left(\left(1-\gamma-x_{\infty}\right) /\left(m+x_{\infty}\right)\right)\right)-(\rho-\delta)$ and determinant $-x_{\infty}(\rho-\delta) \quad\left(-1+\left(\left(1-\gamma-x_{\infty}\right)\right.\right.$ $\left.\left./\left(m+x_{\infty}\right)\right)\right)+\left(\alpha x_{\infty} /\left(m+x_{\infty}\right)\right)\left((\rho-\delta)^{2} / \rho \beta\right)$.

A direct calculation gives

$$
\begin{aligned}
& x_{\infty}\left(-1+\frac{1-\gamma-x_{\infty}}{m+x_{\infty}}\right)-(\rho-\delta) \\
& =\frac{x_{\infty}\left(-m+1-\gamma-2 x_{\infty}\right)-m(\rho-\delta)-x_{\infty}(\rho-\delta)}{m+x_{\infty}} \\
& =\frac{-2 x_{\infty}^{2}-m\left(x_{\infty}+\rho-\delta\right)+(1-\gamma-\rho+\delta) x_{\infty}}{m+x_{\infty}} .
\end{aligned}
$$

(1) From conditions $0<((1-\gamma) / \alpha)<((\rho-\delta) / \rho \beta)$ and $m<\left(\left(-2 x_{\infty}^{2}+(1-\gamma-\rho+\delta) x_{\infty}\right) /\left(x_{\infty}+\rho-\delta\right)\right)$, we obtain

$$
x_{\infty}\left(-1+\frac{1-\gamma-x_{\infty}}{m+x_{\infty}}\right)-(\rho-\delta)>0 .
$$

Therefore, the positive equilibrium $E_{3}$ is unstable.

(2) From conditions $0<((1-\gamma) / \alpha)<((\rho-\delta) / \rho \beta)$ and $m>\left(\left(-2 x_{\infty}^{2}+(1-\gamma-\rho+\delta) x_{\infty}\right) /\left(x_{\infty}+\rho-\delta\right)\right)$, we have

$$
\begin{aligned}
x_{\infty}\left(-1+\frac{1-\gamma-x_{\infty}}{m+x_{\infty}}\right)-(\rho-\delta)<0, & \\
& -x_{\infty}(\rho-\delta)\left(-1+\frac{1-\gamma-x_{\infty}}{m+x_{\infty}}\right)+\frac{\alpha x_{\infty}}{m+x_{\infty}} \frac{(\rho-\delta)^{2}}{\rho \beta} \\
& =-x_{\infty}(\rho-\delta)\left(-1+\frac{1-\gamma}{m+x_{\infty}}-\frac{x_{\infty}}{m+x_{\infty}}-\frac{\alpha(\rho-\delta)}{\rho \beta\left(m+x_{\infty}\right)}\right) \\
& =x_{\infty}(\rho-\delta)\left(1-\frac{1-\gamma}{m+x_{\infty}}+\frac{x_{\infty}}{m+x_{\infty}}+\frac{\alpha(\rho-\delta)}{\rho \beta\left(m+x_{\infty}\right)}\right) \\
& >x_{\infty}(\rho-\delta)\left(1+\frac{x_{\infty}}{m+x_{\infty}}\right)>0 .
\end{aligned}
$$


So, the positive equilibrium $E_{3}$ is locally asymptotically stable.

$$
\begin{aligned}
& \text { Let } \quad \beta(x, y)=(1 / x y), F(x, y)=(\mathrm{d} x \\
& / \mathrm{d} t)=x(1-x)-(\alpha x y / \\
& (m+x))-\gamma x, G(x, y)=(\mathrm{d} y / \mathrm{d} t)=\rho y(1-(\beta y \\
& /(m+x)))-\delta y \text {. By calculations, we have } \\
& \frac{\partial(\beta(x, y) F(x, y))}{\partial x}+\frac{\partial(\beta(x, y) G(x, y))}{\partial y} \\
& =-\frac{1}{y}+\frac{\alpha}{(m+x)^{2}}-\frac{\rho \beta}{x(m+x)} \\
& \leq-\frac{1}{y}+\frac{\alpha}{x(m+x)}-\frac{\rho \beta}{x(m+x)} \\
& <-\frac{1}{y}<0 .
\end{aligned}
$$

Appling Dulac's criterion and Theorem 1, we know that the positive equilibrium $E_{3}$ is globally asymptotically stable.

\section{Local Bifurcation}

hold, then system (5) undergoes a Hopf bifurcation with respect to bifurcation parameter $m$ around the equilibrium point $E_{3}=\left(x_{\infty}, y_{\infty}\right)$. Furthermore, the direction of the Hopf bifurcation is subcritical and the bifurcation periodic solutions are orbitally asymptotically stable if

$$
k_{0}+k_{1}+k_{2}+k_{3}<0 \text {. }
$$

Theorem 7. If $((1-\gamma) / \alpha)=((\rho-\delta) / \rho \beta)>0$, then system (5) undergoes a transcritical bifurcation around $E_{2}$.

Proof. From $((1-\gamma) / \alpha)=((\rho-\delta) / \rho \beta)>0$, we have

$$
\alpha=\frac{(1-\gamma) \rho \beta}{\rho-\delta} \text {. }
$$

Therefore,

$$
f_{\alpha}(x, y)=\left(\begin{array}{c}
-\frac{x y}{m+x} \\
0
\end{array}\right)
$$

We have

$$
f_{\alpha}\left(x_{2}, y_{2}\right)=\left(\begin{array}{l}
0 \\
0
\end{array}\right) \text {. }
$$

The eigenvectors $V$ and $W$ associated to zero eigenvalues of matrixes $J$ and $J^{T}$, respectively, are

$$
\begin{aligned}
& V=\left(\begin{array}{c}
\frac{\rho \beta}{\rho-\delta} \\
1
\end{array}\right), \\
& W=\left(\begin{array}{l}
1 \\
0
\end{array}\right) .
\end{aligned}
$$

So, $W^{T} f_{\alpha}\left(x_{2}, y_{2}\right)=0$.

In addition, $D f_{\alpha}\left(x_{2}, y_{2}\right) V$ equals

$$
D f_{\alpha}\left(x_{2}, y_{2}\right) V=\left(\begin{array}{c}
\frac{\partial(-x y /(m+x))}{\partial x} v_{1}+\frac{\partial(-x y /(m+x))}{\partial y} v_{2} \\
0
\end{array}\right)=\left(\begin{array}{c}
-1 \\
0
\end{array}\right) \text {. }
$$

Combining (33) and (34), we have

$$
W^{T}\left[D f_{\alpha}\left(x_{2}, y_{2}\right) V\right]=\left(\begin{array}{ll}
1 & 0
\end{array}\right)\left(\begin{array}{c}
-1 \\
0
\end{array}\right)=-1 \neq 0 \text {. }
$$

Then, we compute $D^{2} f\left(x_{2}, y_{2}, \alpha\right)(V, V)$ as follows:

$$
D^{2} f\left(x_{2}, y_{2}, \alpha\right)(V, V)=\left(\begin{array}{l}
\frac{\partial^{2} f_{1}}{\partial x^{2}} v_{1} v_{1}+2 \frac{\partial^{2} f_{1}}{\partial x \partial y} v_{1} v_{2}+\frac{\partial^{2} f_{1}}{\partial y^{2}} v_{2} v_{2} \\
\frac{\partial^{2} f_{2}}{\partial x^{2}} v_{1} v_{1}+2 \frac{\partial^{2} f_{2}}{\partial x \partial y} v_{1} v_{2}+\frac{\partial^{2} f_{2}}{\partial y^{2}} v_{2} v_{2}
\end{array}\right)=\left(\begin{array}{c}
-2 \\
2 \rho \beta \\
-\frac{2 \rho}{m}
\end{array}\right)
$$

Combining (33) and (36), we have 


$$
W^{T}\left[D^{2} f_{\alpha}\left(x_{2}, y_{2}\right)(V, V)\right]=\left(\begin{array}{ll}
1 & 0
\end{array}\right)\left(\begin{array}{c}
-2 \\
-\frac{2 \rho \beta}{m}
\end{array}\right)=-2 \neq 0
$$

Since all three conditions of Lemma 2 are satisfied, system (5) undergoes a transcritical bifurcation around $E_{2}$ if $((1-\gamma) / \alpha)=((\rho-\delta) / \rho \beta)>0$.

Let

$$
\begin{aligned}
k_{0}= & \frac{\rho-\delta}{8\left(m+x_{\infty}\right)}\left(\frac{2(m-1)}{m+x_{\infty}}-\frac{3 \alpha m}{\rho \beta\left(m+x_{\infty}\right)^{2}}+\frac{2 \rho \beta}{\alpha}\right), \\
k_{1}= & \frac{1}{8}\left(\frac{m}{x_{\infty}\left(m+x_{\infty}\right)}+\frac{m^{2}(\rho-\delta)}{x_{\infty}\left(m+x_{\infty}\right)^{2}}-\frac{\alpha m^{2}(\rho-\delta)}{\rho \beta x_{\infty}\left(m+x_{\infty}\right)^{3}}\right), \\
k_{2}= & \frac{1}{8} \frac{\rho \beta(\rho-\delta)}{m+x_{\infty}}\left(\frac{2\left(m+x_{\infty}\right)}{\alpha}-\frac{m+2}{\rho \beta}\right)\left(1-\frac{\rho \beta}{\alpha x_{\infty}}+\frac{\alpha m(\rho-\delta)}{\left(m+x_{\infty}\right)^{2}}\left(\frac{m+x_{\infty}}{\alpha}-\frac{1}{\rho \beta}\right)\right. \\
- & \left.\frac{\alpha \rho \beta(\rho-\delta)}{\left(m+x_{\infty}\right)^{2}}\left(\frac{m+x_{\infty}}{\alpha}-\frac{1}{\rho \beta}\right)^{2}\right), \\
k_{3}= & \frac{1}{4}\left(1+\frac{\alpha m(\rho-\delta)}{\left(m+x_{\infty}\right)^{2}}\left(\frac{m+x_{\infty}}{\alpha}-\frac{1}{\rho \beta}\right)\right)\left(1+\frac{\alpha m(\rho-\delta)}{\left(m+x_{\infty}\right)^{2}}\left(\frac{m+x_{\infty}}{\alpha}-\frac{1}{\rho \beta}\right)\right. \\
& \left.-\frac{\alpha m(\rho-\delta)}{\left(m+x_{\infty}\right)^{2}}\left(\frac{m+x_{\infty}}{\alpha}-\frac{1}{\rho \beta}\right)^{2}\right),
\end{aligned}
$$

and $m$ is $m_{H}$ of (40).

Theorem 8. Assume that $0<((1-\gamma) / \alpha)<((\rho-\delta) / \rho \beta)$ is satisfied. If

$$
\begin{gathered}
\rho \beta(1-\gamma)<(2 \alpha-\rho \beta)(\rho-\delta), \\
m_{H}=\frac{-2 x_{\infty}^{2}+(1-\gamma-\rho+\delta) x_{\infty}}{x_{\infty}+\rho-\delta} .
\end{gathered}
$$

The direction of the Hopf bifurcation is supercritical and the bifurcation periodic solutions are unstable if

$$
k_{0}+k_{1}+k_{2}+k_{3}>0
$$

Proof. From (39), we have

$$
2 x_{\infty}<1-\gamma-\rho+\delta .
$$

So, $m_{H}>0$.

The Jacobian matrix of system (5) evaluated at the point $E_{3}$ is given by

$$
J_{E_{3}}=\left(\begin{array}{cc}
x_{\infty}\left(-1+\frac{1-\gamma-x_{\infty}}{m+x_{\infty}}\right) & -\frac{\alpha x_{\infty}}{m+x_{\infty}} \\
\frac{(\rho-\delta)^{2}}{\rho \beta} & -(\rho-\delta)
\end{array}\right) .
$$

The trace $z=\operatorname{Tr}\left(J_{E_{3}}\right)$ and the determinant $D=\operatorname{Det}\left(J_{E_{3}}\right)$ of Jacobian matrix $J_{E_{3}}$ are given by

$$
\begin{aligned}
z & =-x_{\infty}+\frac{x_{\infty}\left(1-\gamma-x_{\infty}\right)}{m+x_{\infty}}-(\rho-\delta) \\
& =\frac{-2 x_{\infty}^{2}+(1-\gamma-m) x_{\infty}}{m+x_{\infty}}-(\rho-\delta) \\
& =\frac{-2 x_{\infty}^{2}+(1-\gamma-\rho+\delta) x_{\infty}-m\left(x_{\infty}+\rho-\delta\right)}{m+x_{\infty}},
\end{aligned}
$$

$$
\begin{aligned}
D & =-x_{\infty}(\rho-\delta)\left(-1+\frac{1-\gamma-x_{\infty}}{m+x_{\infty}}\right)+\frac{(\rho-\delta)^{2}}{\rho \beta} \frac{\alpha x_{\infty}}{m+x_{\infty}} \\
& =x_{\infty}(\rho-\delta) \frac{\rho \beta m-(\rho-\delta) \alpha+(1-\gamma) \rho \beta}{\rho \beta\left(m+x_{\infty}\right)} \\
& =x_{\infty}(\rho-\delta) .
\end{aligned}
$$


By $0<((1-\gamma) / \alpha)<((\rho-\delta) / \rho \beta)$ and (40), we have In addition, we have $\left.z\right|_{m_{H}}=0$ and $\left.D\right|_{m_{H}}>0$.

$$
\begin{aligned}
\frac{\partial z}{\partial m} & =\frac{-\left(x_{\infty}+\rho-\delta\right)\left(m+x_{\infty}\right)-\left(-2 x_{\infty}^{2}+(1-\gamma-\rho+\delta) x_{\infty}-m\left(x_{\infty}+\rho-\delta\right)\right)}{\left(m+x_{\infty}\right)^{2}} \\
& =\frac{x_{\infty}^{2}-(1-\gamma) x_{\infty}}{\left(m+x_{\infty}\right)^{2}} \\
& =-\frac{x_{\infty}\left(1-\gamma-x_{\infty}\right)}{\left(m+x_{\infty}\right)^{2}} \\
& =-\frac{\alpha x_{\infty}(\rho-\delta)}{\rho \beta\left(m+x_{\infty}\right)^{2}} \\
& <0 .
\end{aligned}
$$

So, $\left.(\partial z / \partial m)\right|_{m_{H}}<0$. Therefore, this guarantees the existence of Hopf bifurcation around $E_{3}$.

We translate the equilibrium $E_{3}$ to the origin by the translation $\tilde{x}=x-x_{\infty}$ and $\tilde{y}=y-y_{\infty}$. For the sake of convenience, we still denote $\tilde{x}$ and $\tilde{y}$ by $x$ and $y$, respectively. So, the system (5) becomes

$$
\left\{\begin{array}{l}
\dot{x}=\left(x+x_{\infty}\right)\left(1-x-x_{\infty}\right)-\frac{\alpha\left(x+x_{\infty}\right)\left(y+y_{\infty}\right)}{m+x+x_{\infty}}-\gamma\left(x+x_{\infty}\right), \\
\dot{y}=\rho\left(y+y_{\infty}\right)\left(1-\frac{\beta\left(y+y_{\infty}\right)}{m+x+x_{\infty}}\right)-\delta\left(y+y_{\infty}\right) .
\end{array}\right.
$$

Rewrite system (47) to where

$$
\left(\begin{array}{l}
\dot{x} \\
\dot{y}
\end{array}\right)=J_{E_{3}}\left(\begin{array}{l}
x \\
y
\end{array}\right)+\left(\begin{array}{l}
f_{1}(x, y, m) \\
f_{2}(x, y, m)
\end{array}\right),
$$

$$
\begin{aligned}
f_{1}(x, y, m) & =\left(x+x_{\infty}\right)\left(1-x-x_{\infty}\right)-\frac{\alpha\left(x+x_{\infty}\right)\left(y+y_{\infty}\right)}{m+x+x_{\infty}}-\gamma\left(x+x_{\infty}\right) \\
& -x_{\infty}\left(-1+\frac{1-\gamma-x_{\infty}}{m+x_{\infty}}\right) x+\frac{\alpha x_{\infty}}{m+x_{\infty}} y, \\
f_{2}(x, y, m)= & \rho\left(y+y_{\infty}\right)\left(1-\frac{\beta\left(y+y_{\infty}\right)}{m+x+x_{\infty}}\right)-\delta\left(y+y_{\infty}\right) \\
& -\frac{(\rho-\delta)^{2}}{\rho \beta} x+(\rho-\delta) y .
\end{aligned}
$$

Denote the eigenvalues of $J_{E_{3}}$ by $\varphi+i \omega$ with $\varphi=(z / 2)$ and $\omega=\left(\sqrt{4 D-z^{2}} / 2\right)$.

$$
P=\left(\begin{array}{cc}
1 & 0 \\
N & G
\end{array}\right)
$$

Define matrix 
where $\quad G=\left(\left(\omega\left(m+x_{\infty}\right)\right) / \alpha x_{\infty}\right) \quad$ and $\quad N=(($ $\left.\left.\left(m+x_{\infty}\right)\left(x_{\infty}\left(-1+\left(\left(1-\gamma-x_{\infty}\right) /\left(m+x_{\infty}\right)\right)\right)-\varphi\right)\right) / \alpha x_{\infty}\right)$.

Obviously,

$$
P^{-1}=\left(\begin{array}{cc}
1 & 0 \\
-\frac{N}{G} & \frac{1}{G}
\end{array}\right),
$$
and $\quad \begin{gathered}\text { when } \\ -2 x_{\infty}^{2}+\left(1-\gamma-m_{H}\right) x_{\infty}\end{gathered}=(\rho-\delta)\left(x_{\infty}+m_{H}\right)$, we have

$$
\begin{aligned}
\omega_{0}:=\omega_{m_{H}} & =\sqrt{(\rho-\delta) x_{\infty}}, \\
N_{0}:=\left.N\right|_{m_{H}} & =\frac{\left(m_{H}+x_{\infty}\right)(-1+((1-\gamma)}{\alpha}, \\
& =\frac{\left(m_{H}+x_{\infty}\right)(\rho-\delta)}{\alpha}, \\
\frac{G_{0}}{N_{0}} & :=\frac{\left.(\rho-\delta)\right|_{m_{H}}=\frac{\omega_{0}\left(m_{H}+x_{\infty}\right)}{\alpha x_{\infty}}, \omega_{0},}{\omega_{0}} \\
\frac{1}{G_{0}} & =\frac{\alpha x_{\infty}}{\omega_{0}\left(m_{H}+x_{\infty}\right)} .
\end{aligned}
$$$$
N_{0}:=\left.N\right|_{m_{H}}=\frac{\left(m_{H}+x_{\infty}\right)\left(-1+\left(\left(1-\gamma-x_{\infty}\right) /\left(m_{H}+x_{\infty}\right)\right)\right)}{\alpha}
$$

By the transformation,

$$
\left(\begin{array}{l}
x \\
y
\end{array}\right)=P\left(\begin{array}{l}
u \\
v
\end{array}\right)=\left(\begin{array}{c}
u \\
N u+G v
\end{array}\right)
$$

system (48) becomes

$$
\left(\begin{array}{c}
\dot{u} \\
\dot{v}
\end{array}\right)=\left(\begin{array}{cc}
\varphi & -\omega \\
\omega & \varphi
\end{array}\right)\left(\begin{array}{l}
u \\
v
\end{array}\right)+\left(\begin{array}{l}
F_{1}(u, v, m) \\
F_{2}(u, v, m)
\end{array}\right),
$$

where

$$
\begin{aligned}
F_{1}(u, v, m)= & A_{20} u^{2}+A_{11} u v+A_{21} u^{2} v+A_{30} u^{3} \\
& +o\left(|u|^{4},|u|^{4}|v|\right), \\
F_{2}(u, v, m)= & B_{20} u^{2}+B_{11} u v+B_{02} v^{2}+B_{21} u^{2} v \\
& +o\left(|u|^{4},|u|^{4}|v|\right),
\end{aligned}
$$

with

$$
\begin{aligned}
A_{20}= & -1-\frac{\alpha m\left(N x_{\infty}+N m-y_{\infty}\right)}{\left(m+x_{\infty}\right)^{3}}, \\
A_{11}= & -\frac{\alpha G m}{\left(m+x_{\infty}\right)^{2}}, \\
A_{21}= & \frac{\alpha G m}{(m+x)^{3}}, \\
A_{30}= & \frac{\alpha m\left(N x_{\infty}+N m-y_{\infty}\right)}{\left(m+x_{\infty}\right)^{4}}, \\
B_{20}= & \frac{N}{G}\left(1+\frac{\alpha m\left(N_{\infty}+N m-y_{\infty}\right)}{\left(m+x_{\infty}\right)^{3}}\right) \\
& -\frac{1}{G}\left(\frac{\rho \beta\left(N^{2} x_{\infty}^{2}+2 N^{2} x_{\infty} m-2 N x_{\infty} y_{\infty}\right)+\left(N m-y_{\infty}\right)^{2}}{\left(m+x_{\infty}\right)^{3}}\right), \\
B_{11}= & \frac{\alpha N m}{\left(m+x_{\infty}\right)^{2}}-\frac{2 \rho \beta\left(N x_{\infty}+N m-y_{\infty}\right)}{\left(m+x_{\infty}\right)^{2}}, \\
B_{02}= & -\frac{\rho \beta G}{m+x_{\infty}}, \\
B_{21}= & \frac{2 \rho \beta\left(N x_{\infty}+N m-y_{\infty}\right)-\alpha N m}{\left(m+x_{\infty}\right)^{3}} .
\end{aligned}
$$

In order to determine the stability of the periodic solution, we need to calculate the sign of the coefficient $b\left(m_{H}\right)$, which is given by

$$
\begin{aligned}
b\left(m_{H}\right):= & \frac{1}{16}\left\{F_{u u u}^{1}+F_{u v v}^{1}+F_{u u v}^{2}+F_{v v v}^{2}\right\} \\
& +\frac{1}{16 \omega\left(m_{H}\right)}\left\{F_{u v}^{1}\left(F_{u u}^{1}+F_{v v}^{1}\right)-F_{u v}^{2}\left(F_{u u}^{2}+F_{v v}^{2}\right)\right. \\
& \left.-F_{u u}^{1} F_{u u}^{2}+F_{v v}^{1} F_{v v}^{2}\right\} .
\end{aligned}
$$

where all partial derivatives are evaluated at the bifurcation point $\left(0,0, m_{H}\right)$.

Combining $F^{1}(u, v), F^{2}(u, v), \omega_{0}, G_{0}$, and $N_{0}$, we have 
Discrete Dynamics in Nature and Society

9

$$
\begin{aligned}
& F_{u u u}^{1}(0,0)=\frac{6 m(\rho-\delta)}{\left(m+x_{\infty}\right)^{2}}-\frac{6 \alpha m(\rho-\delta)}{\rho \beta\left(m+x_{\infty}\right)^{3}} \\
& F_{u v v}^{1}(0,0)=0 \\
& F_{u v}^{1}(0,0)=-\frac{m \omega_{0}}{x_{\infty}\left(m+x_{\infty}\right)}, \\
& F_{u u}^{1}(0,0)=-2-2 \alpha m\left(\frac{\rho-\delta}{\alpha\left(m+x_{\infty}\right)}-\frac{\rho-\delta}{\rho \beta\left(m+x_{\infty}\right)^{2}}\right), \\
& F_{v v}^{1}(0,0)=0, \\
& F_{v v v}^{2}(0,0)=0, \\
& F_{u u v}^{2}(0,0)=\frac{4 \rho \beta(\rho-\delta)}{\alpha\left(m+x_{\infty}\right)}-\frac{(2 m+4)(\rho-\delta)}{\left(m+x_{\infty}\right)^{2}}, \\
& F_{u v}^{2}(0,0)=\frac{(m+2)(\rho-\delta)}{\left(m+x_{\infty}\right)}-\frac{2 \rho \beta(\rho-\delta)}{\alpha}, \\
& F_{v v}^{2}(0,0)=-\frac{2 \rho \beta \omega_{0}}{\alpha x_{\infty}}, \\
& F_{u u}^{2}(0,0)=2 \omega_{0}\left(1+\frac{\alpha m(\rho-\delta)}{\left(m+x_{\infty}\right)^{2}}\left(\frac{m+x_{\infty}}{\alpha}-\frac{1}{\rho \beta}\right)-\frac{\alpha \rho \beta(\rho-\delta)}{\left(m+x_{\infty}\right)^{2}}\left(\frac{m+x_{\infty}}{\alpha}-\frac{1}{\rho \beta}\right)^{2}\right)
\end{aligned}
$$

where $m=m_{H}$ and

$$
\begin{aligned}
& b\left(m_{H}\right)= \frac{\rho-\delta}{8\left(m+x_{\infty}\right)}\left(\frac{2(m-1)}{m+x_{\infty}}-\frac{3 \alpha m}{\rho \beta\left(m+x_{\infty}\right)^{2}}+\frac{2 \rho \beta}{\alpha}\right) \\
&+\frac{1}{8}\left(\frac{m}{x_{\infty}\left(m+x_{\infty}\right)}+\frac{m^{2}(\rho-\delta)}{x_{\infty}\left(m+x_{\infty}\right)^{2}}-\frac{\alpha m^{2}(\rho-\delta)}{\rho \beta x_{\infty}\left(m+x_{\infty}\right)^{3}}\right. \\
&+\frac{\rho \beta(\rho-\delta)}{m+x_{\infty}}\left(\frac{2\left(m+x_{\infty}\right)}{\alpha}-\frac{m+2}{\rho \beta}\right) \\
& \times\left(1-\frac{\rho \beta}{\alpha x_{\infty}}+\frac{\alpha m(\rho-\delta)}{\left(m+x_{\infty}\right)^{2}}\left(\frac{m+x_{\infty}}{\alpha}-\frac{1}{\rho \beta}\right)\right. \\
&\left.-\frac{\alpha \rho \beta(\rho-\delta)}{\left(m+x_{\infty}\right)^{2}}\left(\frac{m+x_{\infty}}{\alpha}-\frac{1}{\rho \beta}\right)^{2}\right) \\
&+2\left(1+\frac{\alpha m(\rho-\delta)}{\left(m+x_{\infty}\right)^{2}}\left(\frac{m+x_{\infty}}{\alpha}-\frac{1}{\rho \beta}\right)\right)\left(1+\frac{\alpha m(\rho-\delta)}{\left(m+x_{\infty}\right)^{2}}\left(\frac{m+x_{\infty}}{\alpha}-\frac{1}{\rho \beta}\right)\right. \\
&= k_{0}+k_{1}+k_{2}+k_{3} . \\
&\left(m+x_{\infty}\right)^{2} \\
&\left(\frac{m-\delta)}{\alpha}\left(\frac{m+x_{\infty}}{\rho \beta}\right)^{2}\right)
\end{aligned}
$$




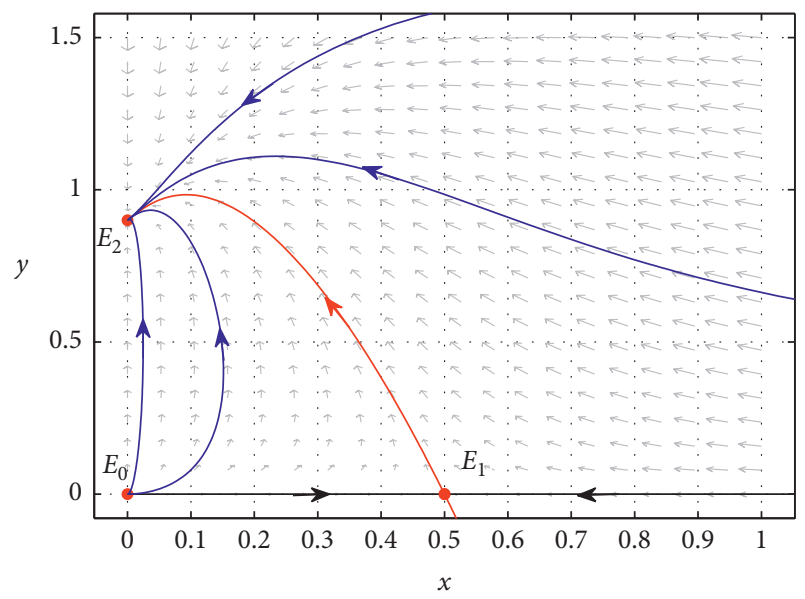

Figure 3: Phase portrait for system (12) with $\alpha=\beta=\rho=m=1$, $\gamma=0.5$, and $\delta=0.1$.

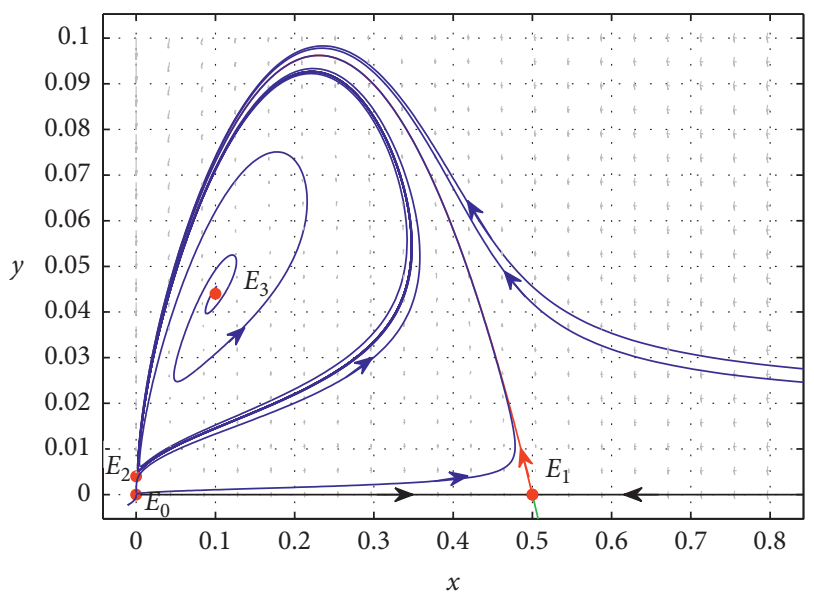

Figure 4: Phase portrait for system (12) with $\alpha=\rho=1, \beta=0.5$, $m=0.01, \gamma=0.5$, and $\delta=0.8$.

Combining (41), we have $b\left(m_{H}\right)<0$. Therefore, according to Poincare-Andronow's Hopf bifurcation theory, we have the direction of the Hopf bifurcation is subcritical and the bifurcation periodic solutions are orbitally asymptotically stable.

In addition, combining (42), we have $b\left(m_{H}\right)>0$. Therefore, according to Poincare-Andronow's Hopf bifurcation theory, we have the direction of the Hopf bifurcation is supercritical and the bifurcation periodic solutions are unstable.

\section{Numerical Illustrations}

In this section, we perform numerical simulations about system (5). Figure 3 shows that $E_{0}$ is an unstable node point, $E_{1}$ is a saddle point, $E_{3}$ does not exist, and $E_{2}$ is asymptotically stable and every orbit tends to it. Figure 4 shows that $E_{0}$ is an unstable node point, $E_{1}$ is a saddle point, $E_{2}$ is unstable, $E_{3}$ is unstable, and there is a limit cycle around $E_{3}$ to which every orbit tends. Figure 5 shows that $E_{0}$ is an unstable node point, $E_{1}$ is unstable and $E_{2}$ is also unstable, but $E_{3}$ is asymptotically stable and every orbit approaches this equilibrium.

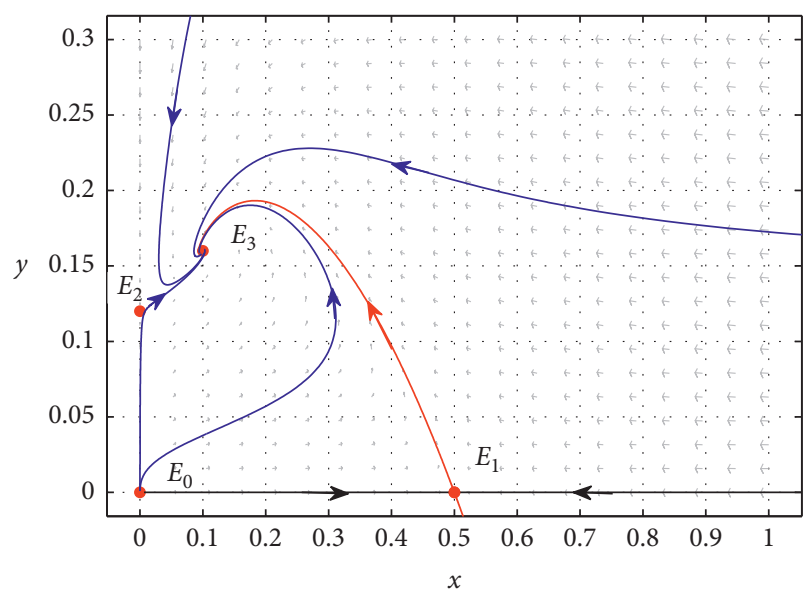

FIgURE 5: Phase portrait for system (12) with $\alpha=\rho=1, \beta=0.5$, $m=0.3, \gamma=0.5$, and $\delta=0.8$.

\section{Data Availability}

No data were used to support this study.

\section{Conflicts of Interest}

The authors declare that they have no conflicts of interest.

\section{Acknowledgments}

This work was partially supported by National Natural Science Foundation of P.R.China (no. 11661037).

\section{References}

[1] N. F. Britton, Essential Mathematical Biology, Springer, Berlin, Germany, 2013.

[2] J. D. Murray, Mathematical Biology I: An Introduction, Springer, Berlin, Germany, 2002.

[3] J. D. Murray, Mathematical Biology: Spatial Models and Biomedical Applications, Springer, Berlin, Germany, 2002.

[4] F. Brauer and C. Castillo-Chavez, Mathematical Models in Population Biology and Epidemiology, Springer, Berlin, Germany, 2011.

[5] W. Yang and Y. Li, "Dynamics of a diffusive predator-prey model with modified Leslie-Gower and Holling-type III schemes," Computers \& Mathematics with Applications, vol. 65, no. 11, pp. 1727-1737, 2013.

[6] F. Wu and Y. Jiao, "Stability and Hopf bifurcation of a predator-prey model," Boundary Value Problems, vol. 2019, 2019.

[7] J. Huang, S. Ruan, and J. Song, "Bifurcations in a predatorprey system of Leslie type with generalized Holling type III functional response," Journal of Differential Equations, vol. 257, no. 6, pp. 1721-1752, 2014.

[8] L. Chen and F. Chen, "Global stability of a Leslie-Gower predator-prey model with feedback controls," Applied Mathematics Letters, vol. 22, no. 9, pp. 1330-1334, 2009.

[9] G. Lin, J. Ji, L. Wang, and J. Yu, "Multitype bistability and long transients in a delayed spruce budworm population model," Journal of Differential Equations, vol. 283, pp. 263-289, 2021. 
[10] N. Mart-Jeraldo and P. Aguirre, "Allee effect acting on the prey species in a Leslie-Gower predation model," Nonlinear Analysis: Real World Applications, vol. 45, pp. 895-917, 2019.

[11] M. Aziz-Aloui and M. Daher Okiye, "Boundedness and global stability for a predator-prey model with modified LeslieGower and holling-type II schemes," Applied Mathematics Letters, vol. 16, pp. 1069-1075, 2003.

[12] C. Lin and C. Ho, "Local and global stability for a predatorprey model of modified Leslie-Gower and holling-type II with time-delay," Tunghai Science, vol. 8, pp. 33-61, 2006.

[13] J. Huang, Y. Gong, Y. Gong, and S. Ruan, "Bifurcation analysis in a predator-prey model with constant-yield predator harvesting," Discrete \& Continuous Dynamical Systems- $B$, vol. 18, no. 8, pp. 2101-2121, 2013.

[14] S. Chakraborty, S. Pal, and N. Bairagi, "Predator-prey interaction with harvesting: mathematical study with biological ramifications," Applied Mathematical Modelling, vol. 36, no. 9, pp. 4044-4059, 2012.

[15] P. Lenzini and J. Rebaza, "Nonconstant predator harvesting on ratio-dependent predator-prey models," Applied Mathematical Sciences, vol. 4, pp. 791-803, 2010.

[16] J. Ali, R. Shivaji, and K. Wampler, "Population models with diffusion, strong Allee effect and constant yield harvesting," Journal of Mathematical Analysis and Applications, vol. 352, no. 2, pp. 907-913, 2009.

[17] C. Zhu and K. Lan, "Phase portraits, "Hopf bifurcations and limit cycles of Leslie-Gower predator-prey systems with harvesting rates"' Discrete and Continuous Dynamical Systems Series B, vol. 14, pp. 289-306, 2010.

[18] R. P. Gupta and P. Chandra, "Bifurcation analysis of modified Leslie-Gower predator-prey model with michaelis-menten type prey harvesting," Journal of Mathematical Analysis and Applications, vol. 398, no. 1, pp. 278-295, 2013.

[19] F. Chen, "On a nonlinear nonautonomous predator-prey model with diffusion and distributed delay," Journal of Computational and Applied Mathematics, vol. 180, no. 1, pp. 33-49, 2005.

[20] L. Peko, Differential Equations and Dynamical Systems, Springer, Berlin, Germany, 2001.

[21] B. Pirayesh, A. Pazirandeh, and M. Akbari, "Local bifurcation analysis in nuclear reactor dynamics by Sotomayor's theorem," Annals of Nuclear Energy, vol. 94, pp. 716-731, 2016. 